\title{
Characterization and Mapping of Leaf Rust and Stripe Rust Resistance Loci in Hexaploid Wheat Lines UC1110 and PI610750 under Mexican Environments
}

\begin{abstract}
Caixia Lan', lago L. Hale², Sybil A. Herrera-Foessel', Bhoja R. Basnet', Mandeep S. Randhawa ${ }^{1}$, Julio Huerta-Espino ${ }^{3}$, Jorge Dubcovsky ${ }^{4}$ and Ravi P. Singh ${ }^{1 *}$

${ }^{1}$ International Maize and Wheat Improvement Center, Mexico City, Mexico, ${ }^{2}$ Department of Biological Sciences, University of New Hampshire, Durham, NH, United States, ${ }^{3}$ Campo Experimental Valle de Mexico, Instituto Nacional de Investigaciones Forestales, Agrícolas y Pecuarias, Chapingo, Mexico, ${ }^{4}$ Department of Plant Sciences, University of California, Davis, Davis, CA, United States
\end{abstract}

OPEN ACCESS

Edited by:

Luigi Cattivelli,

Consiglio per la ricerca in agricoltura e l'analisi dell'economia agraria (CREA),

Italy

Reviewed by: Anna Maria Mastrangelo,

Centro di Ricerca per l'Orticoltura

(CRA), Italy

Morten Lillemo,

Norwegian University of Life Sciences,

Norway

*Correspondence:

Ravi P. Singh

r.singh@cgiar.org

Specialty section: This article was submitted to Crop Science and Horticulture, a section of the journal

Frontiers in Plant Science

Received: 19 May 2017 Accepted: 04 August 2017 Published: 21 August 2017

Citation:

Lan C, Hale IL, Herrera-Foessel SA, Basnet BR, Randhawa MS,

Huerta-Espino J, Dubcovsky J and

Singh RP (2017) Characterization and Mapping of Leaf Rust and Stripe

Rust Resistance Loci in Hexaploid Wheat Lines UC1110 and PI610750 under Mexican Environments.

Front. Plant Sci. 8:1450. doi: 10.3389/fpls.2017.01450
Growing resistant wheat varieties is a key method of minimizing the extent of yield losses caused by the globally important wheat leaf rust (LR) and stripe rust (YR) diseases. In this study, a population of $186 \mathrm{~F}_{8}$ recombinant inbred lines (RILs) derived from a cross between a synthetic wheat derivative (PI610750) and an adapted common wheat line (cv. "UC1110") were phenotyped for LR and YR response at both seedling and adult plant stages over multiple seasons. Using a genetic linkage map consisting of single sequence repeats and diversity arrays technology markers, in combination with inclusive composite interval mapping analysis, we detected a new LR adult plant resistance (APR) locus, QLr.cim-2DS, contributed by UC1110. One co-located resistance locus to both rusts, QLr.cim-3DC/QYr.cim-3DC, and the known seedling resistance gene Lr26 were also mapped. QLr.cim-2DS and QLr.cim-3DC showed a marginally significant interaction for $L R$ resistance in the adult plant stage. In addition, two previously reported YR APR loci, QYr.ucw-3BS and Yr48, were found to exhibit stable performances in rust environments in both Mexico and the United States and showed a highly significant interaction in the field. Yr48 was also observed to confer intermediate seedling resistance against Mexican $Y R$ races, thus suggesting it should be re-classified as an allstage resistance gene. We also identified 5 and 2 RILs that possessed all detected YR and LR resistance loci, respectively. With the closely linked molecular markers reported here, these RILs could be used as donors for multiple resistance loci to both rusts in wheat breeding programs.

Keywords: adult plant resistance, durable rust resistance, leaf rust, stripe rust, wheat

\section{INTRODUCTION}

Wheat leaf rust (LR) and stripe rust (YR), caused by the air-borne fungi Puccinia triticina $(P t)$ and Puccinia striiformis f. sp. tritici (Pst), respectively, are considered the primary biotic threats to wheat production globally (Todorovska et al., 2009). LR can result in $20-40 \%$ yield losses resulting in dying leaves, reduced floret set, and grain shriveling (Knott and Padidam, 1988); and the potential 
yield loss for YR-susceptible cultivars was determined to be more than $60 \%$ in the Pacific Northwest (Chen, 2014). Recently, Hovmøller et al. (2015) found that the highly aggressive and heat-tolerant "Warrior" and "Kranich" races of YR originated from a sexually recombining population in the center of diversity of the YR fungus near the Himalayan region of central Asia. A new variant of the aggressive YR race group common in North America (Chen, 2005) caused a widespread epidemic in the highlands of Mexico in 2014 on wheat variety "Nana F2007" due to its combination of virulence on resistance genes Yr3, Yr27, and Yr31 (Huerta-Espino et al., 2015). Breeding for durable resistance to wheat rusts continues to be a global priority but is also a challenge due to the complexity of interactions among resistance genes as well as the wide diversity and continuous evolution of pathogen races (Lowe et al., 2011a; Vazquez et al., 2015).

Genetic resistance to rust pathogens is commonly characterized as belonging to one of the following three general categories: (1) race-specific seedling resistance, also known as all-stage resistance (Chen, 2013); (2) race-specific adult plant resistance (APR); and (3) race non-specific APR, also known as slow-rusting or partial resistance (Johnson and Law, 1973; Das et al., 1992). Several all-stage, race-specific resistance genes characterized in wheat are known to be associated with various degrees of hypersensitive response (McIntosh et al., 2016) through elicitation of programmed cell death in the host if the pathogen possesses the corresponding avirulence genes (Flor, 1942). Some race-specific APR genes are known for both YR (e.g., Yr11, Yr12, Yr13, Yr14, Yr16, Yr39, and Yr49) and LR (e.g., Lr13, Lr22a, and Lr22b) (Seyfarth, 2000; McIntosh et al., 2016). These two race-specific resistances are frequently non-durable under high selection pressure on the pathogen due to rapid mutation rates and dependence on a direct protein-protein interaction for recognition (Jones and Dangl, 2006). In contrast, race non-specific APR genes generally do not provide a high level of resistance in isolation, but can do so when deployed in combination with other minor genes (Singh et al., 2000a). To date, more than $76 \mathrm{LR}$ and 78 YR resistance genes have been cataloged (McIntosh et al., 2016). Of these, only Lr34/Yr18/Pm38/Sr57 (Singh et al., 2012), Lr46/Yr29/Pm39/Sr58 (Singh et al., 2013), and Lr67/Yr46/Pm46/Sr55 (Herrera-Foessel et al., 2014) confer pleiotropic, race non-specific APR to LR, YR, powdery mildew, and stem rust. The molecular structures of Lr34/Yr18 (Krattinger et al., 2009) and Lr67/Yr46 (Moore et al., 2015) indicate different underlying mechanisms of the transporter-mediated APR conferred by these genes.

Over the past 20 years, more than 50 quantitative trait loci (QTL) for LR and YR resistance have been reported using a wide range of markers, including diversity arrays technology (DArT), single sequence repeats (SSRs), and single-nucleotide polymorphisms (Rosewarne et al., 2013; Li et al., 2014; Maccaferri et al., 2015); and the genetic locations of these QTL are continually being refined through subsequent mapping studies. In a previous study, Lowe et al. (2011b) reported moderate levels of field resistance to YR in both the California (CA)-adapted hexaploid wheat breeding line UC1110 and the synthetic wheat derivative PI610750. Using a population of 186 recombinant inbred lines (RILs) derived from a cross between these parents, four YR resistance QTL (two from UC1110 and two from PI610750) were identified and characterized under the CA rust environment. The objectives of this follow-up study were to: (1) investigate the performance of these previously reported YR resistance loci in the Mexican rust environment; (2) identify potential sources of both seedling resistance and race non-specific APR to both LR and YR in this mapping population; and (3) Determine interactions between the identified resistance loci in the adult plant stage.

\section{MATERIALS AND METHODS}

\section{Plant Materials}

A population of $186 \quad \mathrm{~F}_{7}$-derived $\mathrm{F}_{8} \quad$ RILs was developed from a cross between UC1110 (pedigree: Chukar/3/Yding//Bluebird/Chanate) and PI610750 [pedigree: Croc1/Aegilops tauschii (Synthetic 205)//Kauz] (Lowe et al., 2011b). UC1110, an adapted Californian hard white spring wheat line bred by and used as a parent in the University of California, Davis (UC Davis) wheat breeding program, carries YR resistance loci on wheat chromosomes 2BS and 3BS (Lowe et al., 2011b) under the CA rust environment. PI610750, a synthetic derivative spring wheat line developed by CIMMYT in Mexico, carries the YR APR gene Yr48 and a minor YR resistance locus on chromosome 2AS (Lowe et al., 2011b). After multiplication of the RILs and parents in Mexico, $\mathrm{F}_{8}$ seeds were used for all genotyping and disease phenotyping.

\section{Greenhouse Experiments (Seedling Stage) Leaf Rust}

The two parents and 186 RILs were tested in January 2014 under greenhouse conditions with $P$. triticina race MBJ/SP [avirulence/virulence formula: $\operatorname{Lr} 2 a, 2 b, 2 c, 3 k a, 9,16,18,19$, $21,24,25,(26), 28,29,30,32,33,36 / 1,3,3 b g, 10,11$, $13,15,17,20,23,27+31$ (Lan et al., 2014b)]. A set of 48 differential lines with known LR resistance genes (most in the Thatcher background, including Thatcher + Lr26) were included in the seedling tests to compare the infection types (ITs) of the parents and RILs to those associated with known resistance genes. All RILs were sown in trays in hills of six to eight seeds each (48 lines/tray). Once seedlings reached the twoleaf stage, they were inoculated by spraying urediniospores suspended in lightweight Soltrol 170 mineral oil using an atomizer. To facilitate evaporation of the oil, the inoculated plants were left on an open bench for $20 \mathrm{~min}$ before being transferred into an $18^{\circ} \mathrm{C}$ dew chamber overnight, then back to the greenhouse. Based on a monitor that measured air temperature in the greenhouse every $30 \mathrm{~min}$, the minimum, maximum, and mean post-inoculation temperatures were 10.2, 25.8 , and $20.0^{\circ} \mathrm{C}$, respectively. LR ITs were recorded 11 days post-inoculation based on the following scale modified from Roelfs et al. (1992): "0" = no visible symptoms; ";" = only necrotic/chlorotic flecks without any uredinia; "1" $=$ small uredinia surrounded by necrosis; " 2 " = small to medium uredinia 
surrounded by chlorosis or necrosis; " 3 " = medium-sized uredinia without chlorosis or necrosis; " 4 " = large-sized uredinia without chlorosis or necrosis; " $\mathrm{X}$ " = random distribution of variable-sized uredinia; "3C3" = medium-sized uredinia with chlorosis; "; $11+"=$ necrotic/chlorotic flecks with small to medium uredinia; and "+" and "-" were used when uredinia were somewhat larger or smaller than normal for the ITs. ITs of 3 , $3+$, and 4 are considered to be susceptible host reactions, whereas all of other ITs are considered resistant.

\section{Stripe Rust}

In January of 2016 and 2017, the parents and RILs were evaluated for their reaction to YR at the seedling stage using the recent Mexican $P$. striiformis isolate Mex14.191 virulent on $Y r$ genes 2, 3, 6, 7, 8, 9, 17, 27, 31, and $A$ (HuertaEspino et al., 2015). A total of 30 differential lines with known YR resistance genes (mostly in Avocet background) were also included. The inoculation method was the same as described above for LR, but with a lower incubation temperature in the dew chamber of $7^{\circ} \mathrm{C}$. Inoculated plants were placed in the dew chamber for $24 \mathrm{~h}$ and then transferred to the greenhouse. The minimum, maximum, and mean postinoculation greenhouse temperatures were $7.4,27.5$, and $14.9^{\circ} \mathrm{C}$, respectively. ITs were recorded approximately 2 weeks postinoculation, based on a 0-9 scale modified from McNeal et al. (1971), where "0" = no visible infection; "1" = necrotic/chlorotic flecks without sporulation; " 2 " = necrotic/chlorotic stripes without sporulation; " 3 " $=$ necrotic/chlorotic stripes with trace sporulation; " 4 " = necrotic/chlorotic stripes with light sporulation; " 5 " = necrotic/chlorotic stripes with intermediate sporulation; " 56 " = necrotic/chlorotic stripes with intermediate to moderate sporulation; " 6 " $=$ chlorotic stripes with moderate sporulation; " 7 " = stripes without chlorosis or necrosis and with moderate sporulation; " 8 " $=$ stripes without chlorosis or necrosis and with sufficient sporulation; and " 9 " = stripes without chlorosis or necrosis and abundant sporulation. ITs of 0-4, 5-6, and 7-9 are categorized as resistant, intermediate, and susceptible, respectively.

\section{Field Experiments (Adult Plant Stage)}

The parents and RIL population were evaluated for APR to LR at Ciudad Obregón in the Yaqui Valley in northern Mexico during the 2011-2012 growing season (LR2012Y) and at El Batán, CIMMYT headquarters outside Mexico City, in the 2012 and 2013 seasons (LR2012B and LR2013B). Similarly, two YR experiments were conducted at another CIMMYT research station in Toluca, Mexico, during the 2012 and 2013 growing seasons (YR2012 and YR2013). Field plots consisted of 0.7-m paired rows planted with approximately 60 plants of each line. The Avocet near-isoline for $Y r 24 / 26$ was used as the LR spreader, whereas a mixture of susceptible wheat lines comprised of cv. "Morocco," an Avocet near-isoline for gene Yr31, and six lines derived from an Avocet/Attila cross, was used as the YR spreader in field trials. The spreaders were planted around the experimental area and as hill plots in the middle of a 0.3-m pathway on one side of each experimental plot. The LR epidemic was initiated by spraying the LR spreader with an equal mixture of $P$. triticina races MBJ/SP and MCJ/SP suspended in Soltrol 170. The main difference between the two races is partial (MBJ/SP) and complete (MCJ/SP) virulence on Lr26 (Lan et al., 2014b). For the YR trials, a mixture of $P$. striiformis races Mex96.11 and Mex08.13 was sprayed onto the spreaders within and around the experimental areas. Isolate Mex08.13 belongs to the lineage of the aggressive $\mathrm{YR}$ race that was first detected in California (Chen, 2005) and is virulent on $Y r$ genes 2, 6, 7, 8, 9, 31, and $A$, whereas Mex96.11 is virulent on $Y r$ genes 2, 3, 6, 7, 9, 27, and $A$ (Lan et al., 2014b). Disease severities (DS) for the parents and the RILs were recorded three times in each experiment, according to the modified Cobb's scale (Peterson et al., 1948) and host response to infection was determined according to Roelfs et al. (1992), where " $\mathrm{R}$ " = resistant, or miniature uredinia surrounded by necrotic tissues; "MR" = moderately resistant, or smaller to moderate-sized uredinia surrounded by necrotic or chlorotic tissues; "MS" = moderately susceptible, or moderate-sized uredinia without necrotic or chlorotic tissues; and " $\mathrm{S}$ " = susceptible, or large uredinia without necrotic or chlorotic tissues. In cases of repeated DS data, the first note was recorded when the susceptible check Avocet displayed approximately $80 \%$ severity and repeated about a week later when it reached $90-100 \%$. For multiple disease readings, the area under the disease progress curve (AUDPC) was calculated using the method described by Bjarko and Line (1988). Final DS (FDS) and AUDPC scores were used in downstream genetic analyses.

\section{Molecular Markers, Linkage Map Construction, and QTL Analysis}

In this study, baseline molecular marker data and linkage map information for the UC1110/PI610750 population was used from a previous study by Lowe et al. (2011b). Linkage maps were graphically produced by using MapChart (Voorrips, 2002). QTL mapping of FDS and AUDPC for each experiment was carried out using inclusive composite interval mapping (ICIM) 4.1 (Meng et al., 2015) The ICIM method was also used to analyze the final mean DS for each disease (LRM and YRM) across experiments. The logarithm of odds (LOD) threshold to declare a QTL significant at $P=0.05$ for each trait was determined based on 1,000 permutation tests. Stepwise regression was used to estimate both the main effects and percentages of phenotypic variance explained (PVE) by individual QTL (at LOD peaks). The QTL result was confirmed by QTL Cartographer (Wang et al., 2012). The seedling resistance gene was mapped by the Joinmap 4.1 software (van Ooijen, 2006). Marker orders and chromosomal assignments of linkage groups were verified using wheat SSR consensus genetic maps and the physical bin locations of DArT markers (Somers et al., 2004; Francki et al., 2009; Huang et al., 2012; Wilkinson et al., 2012). QTL designations were assigned following recommended practices ${ }^{1}$. Factorial analyses of variance (ANOVA) were conducted to test for the significances of interactions between detected resistance loci based on AUDPC.

${ }^{1}$ https://wheat.pw.usda.gov/GG2/index.shtml 


\section{RESULTS}

\section{Seedling Responses to LR and YR Leaf Rust}

Seedling ITs were ";11+" and "3C3" for P1610750 and UC1110, respectively, against $P$. triticina race $\mathrm{MBJ} / \mathrm{SP}$ (data not shown). The seedling resistance contributed by PI610750 was mapped to wheat chromosome $1 \mathrm{~B}, 23.2 \mathrm{cM}$ proximal of a molecular marker cluster comprised of two SSR and 173 DArT markers (Supplementary Table S1). This line displayed a similar IT to the Lr26 tester (1RS.1BL translocation), indicating that Lr26 may be the gene conferring seedling resistance to $L R$ race MBJ/SP in PI610750. This is also supported by the presence of the 1RS.1BL translocation in PI610750, its source most likely being one of its parents, cv. "Kauz" (Lowe et al., 2011b).

\section{Stripe Rust}

The ITs of PI610750 and UC1110 were " 56 " and "6," respectively, against $P$. striiformis isolate Mex14.191 at the seedling stage. The seedling resistance gene from PI610750 was mapped to the proximal end of chromosome 5AL, where it co-segregated with molecular markers gwm410 and wPt-9800 (Figure 1). This chromosome position coincides with the location of $\operatorname{Yr} 48$ reported in the previous study by Lowe et al. (2011b), thus indicating that Yr48 confers an intermediate level of seedling resistance to race Mex14.191. The seedling YR IT ranged from 4 to 6 for RILs carrying Yr48, whereas it was 7 to 8 for RILs lacking this resistance gene (Figure 2A). Mean YR DS ranged from 1 to $60 \%$ for RILs carrying Yr48, whereas it was 1 to $100 \%$ for RILs without it (Figure 2B). Overall, 125 RILs exhibited ITs ranging from 4 to 6 (resistant), while 57 RILs exhibited ITs ranging from 7 to 8 (susceptible), a result which indicates a distorted distribution for this gene in the population $(P<0.0001)$. Similar distortion (i.e., 122-129 RILs carrying the PI610750 allele and 57-63 RILs carrying the UC1110 allele $(P<0.0001)$ was observed for the five molecular markers most closely linked to Yr48 (Supplementary Table S2).

\section{Adult Plant Responses to LR and YR}

The FDS for LR (LRM) were $1 \mathrm{MR}$ for PI610750 and 15 MSS, 20 MSS, and 30 MSS for UC1110 in LR2012Y, LR2012B, and LR2013B, respectively, over the three seasons of this study (Figures 3A-C); and LRM for the RILs ranged from 18 to $41 \%$ in those same environments (data not shown). LR severities among the RILs was continually distributed across 3 years (Figure 3), indicating quantitative inheritance of APR to LR in the population.

For YR, the FDS (YRMs) for PI610750 were $5 \mathrm{MR}$ and for UC1110 were $30 \mathrm{M}$ and $40 \mathrm{M}$ in YR2012 and YR2013, respectively (Figures 4A,B). YRM for the RILs ranged between 14 and 30\% across the two seasons (Figure 4). The frequency distributions of YR severity among the RILs showed a bimodal distribution in both environments (Figure 4), which is an indication for the segregation of a major seedling resistance gene in the UC1110/PI610750 population.

Pearson correlation coefficients $(r)$ of LR severity ranged from 0.57 to 0.81 among the three environments, whereas it

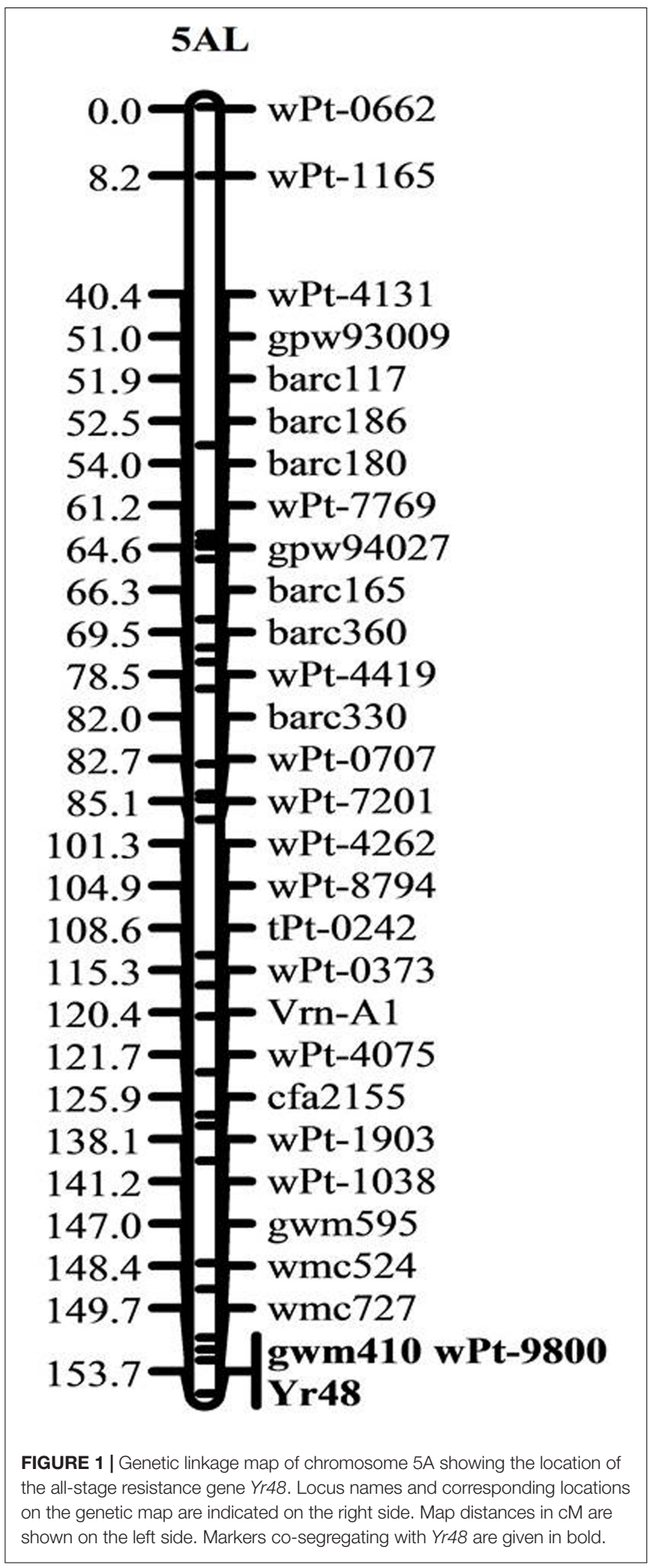

was 0.66 for YR severity over the 2 years (Table 1). Low and non-significant correlations were observed between LR and YR severities ( $r=0.03-0.25)$ across all environments. 

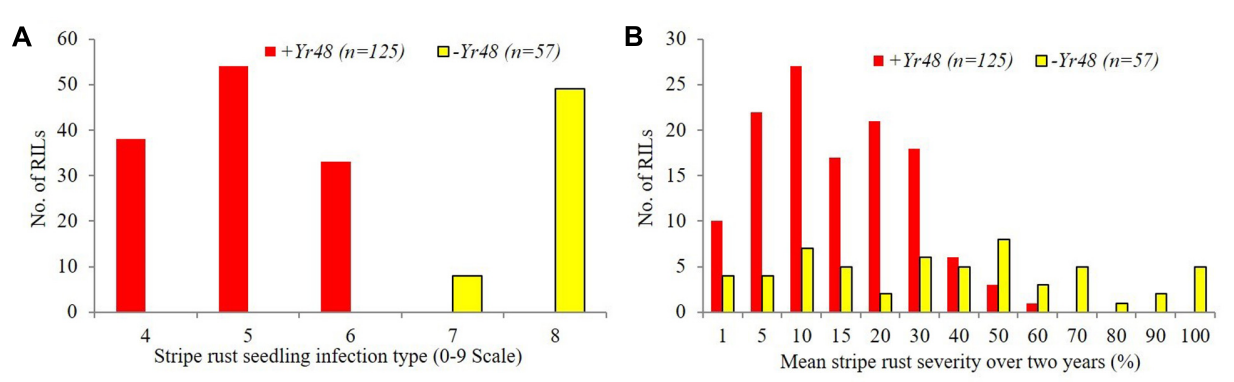

FIGURE 2 | Frequency distributions of stripe rust response for UC1110/PI610750 recombinant inbred lines (RILs) possessing and lacking Yr48 at the seedling stage (A) and the adult plant stage (B).
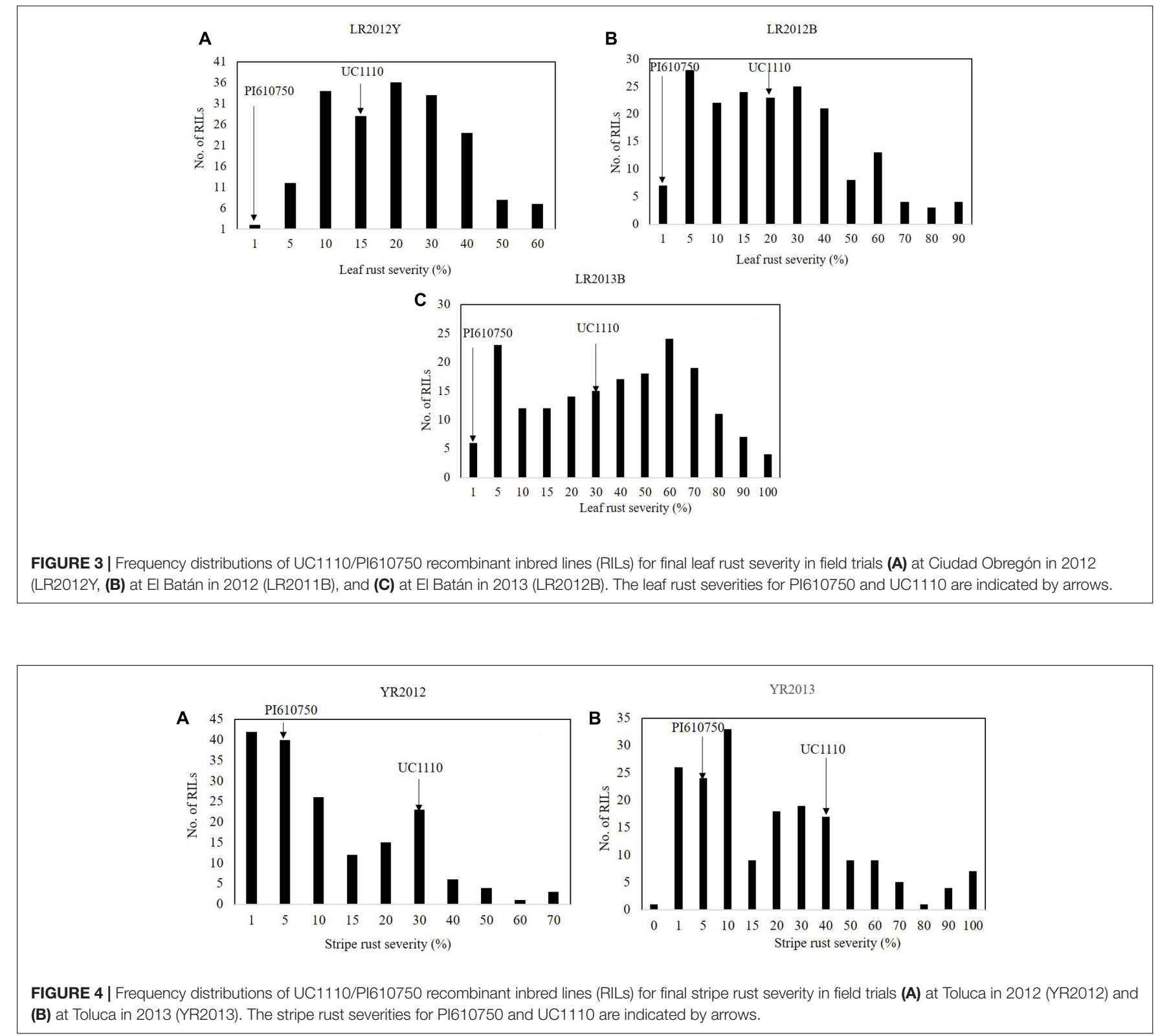
TABLE 1 | Pearson's correlation coefficients (r) among the UC1110/PI610750 RILs for final leaf rust severities in three environments (Ciudad Obregón LR2012Y, El Batán LR2012B and LR2013B) and stripe rust severities in two (Toluca YR2012 and YR2013) environments in Mexico.

\begin{tabular}{lcccc}
\hline Environment & LR2012Y & LR2012B & LR2013B & YR2012 \\
\hline LR2012B & $0.57^{* *}$ & & & \\
LR2013B & $0.58^{* *}$ & $0.81^{* *}$ & & \\
YR2012 & 0.24 & 0.18 & 0.25 & \\
YR2013 & 0.23 & 0.03 & 0.14 & $0.66^{* *}$ \\
\hline
\end{tabular}

$* * P<0.0001$

\section{APR Locus to LR on Chromosome 2DS}

A major QTL for LR resistance, QLr.cim-2DS, was located on the end of the short arm of chromosome 2D and interval flanked by SSR markers $c f d 51$ and $g w m 455$ (Figure 5A). This QTL was consistently detected in all three LR field experiments and explained 11.8-26.6\% of the observed variation in LRM (Table 2). Mean LR severities for RILs carrying QLr.cim-2DS ranged from 1 to $50 \%$ (with an exception of entry 86 with 70\% LR FDS), whereas it was 5 to $90 \%$ for RILs without this locus (Figure 6). This resistance locus derives from UC1110 (Table 2).

\section{Co-located APR Loci to LR and YR on Chromosome 3D}

Co-located APR loci to both rusts, QLr.cim-3DC/QYr.cim-3DC, were mapped near the centromeric region of chromosome $3 \mathrm{D}$ (3DC), flanked by SSR markers gwm341 and barc1119 (Table 2 and Figure 5B). This co-located resistance locus was also contributed by UC1110 and explained 23.3 and $6.1 \%$ of the observed variation in YRM and LRM, respectively (Table 2).
In addition, the previous study reported YR resistance loci on chromosome 3BS and Yr48 were also identified in the present study under Mexican rust environments (Table 2).

\section{Interactions between Resistance Loci for LR and YR}

The $\mathrm{F}_{8}$ RILs were divided into groups based on the markerinferred factorial combinations of the two LR resistance QTL and three YR resistance QTL (Tables 3, 4). Specifically, the flanking molecular markers for each QTL were used to infer the presence of parental alleles in each RIL. A marginally significant interaction $(P=0.037)$ between QLr.cim-2DS and QLr.cim-3DC on LR was observed across 3 years based on AUDPC (Table 3), which only explained $1.4 \%$ of LR AUDPC variation. QYr.ucw-3BS did not show a big effect on YR resistance at the adult plant stage when it was present alone in the adult plant stage (2.8\% of the variation), but it showed a highly significant interaction with $\operatorname{Yr} 48$ that explained $37.9 \%$ of the variation (Table 4). By contrast, no significant gene interaction between QYr.ucw-3BS $\times$ QYr.cim-3DC, QYr.cim-3DC $\times$ Yr48, and QYr.cuw-3BS $\times$ Yr48 $\times$ QYr.cim-3DC was detected, indicating additive effects was present between them. RILs combing all of three YR resistance QTL displayed severity from 0.1 to $3.6 \%$ with a near immune response to YR.

\section{DISCUSSION}

UC1110 showed a moderate level of APR to LR and YR under Mexican rust environments, whereas PI610750 remained highly resistant to both rusts at the adult plant stage and showed an
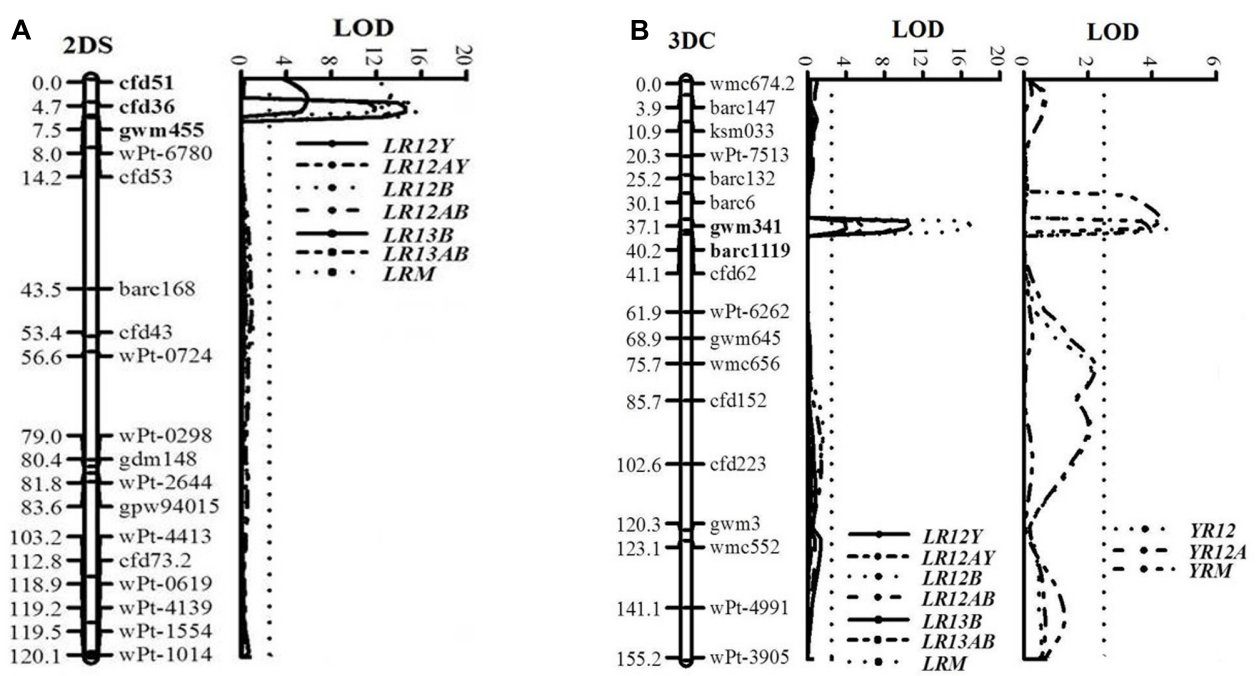

FIGURE 5 | LOD plots of quantitative trait loci (QTL) for adult plant resistance to leaf rust on chromosomes 2DS (A) and to both leaf and stripe rust on chromosome 3DC (B), identified using IciMapping 4.1 in the UC1110/PI610750 RIL population. Significant LOD were detected based on 1,000 permutations. Positions (cM) of the molecular markers on chromosomes are shown on the vertical axes; cumulative genetic distances of linkage groups are also shown. LR12Y, LR12B, and LR13B: leaf rust phenotypic data at Ciudad Obregón in 2012 and El Batán during 2012 and 2013, respectively; YR12: stripe rust phenotypic data at Toluca in 2012; LR12AY, LR12AB, LR13AB, and YR12A: area under the disease progress curve (AUDPC); LRM and YRM: mean final disease severity over test environments. QTL flanking markers are in bold. 
TABLE 2 | Quantitative trait loci (QTL) detected in the UC1110/PI610750 RIL population for adult plant resistance to leaf rust (Ciudad Obregón LR2012Y, El Batán LR2012B and LR2013B) and stripe rust (Toluca YR2012 and YR2013); traits include the area under the disease progress curve (AUDPC, viz. LR2012AY) and the mean of final disease severity over all environment (LRM and YRM).

\begin{tabular}{|c|c|c|c|c|c|c|c|c|}
\hline QTL/gene $^{a}$ & Trait name & Position & Left marker & Right marker & LOD & PVE (\%) & Add & Resistance source \\
\hline \multirow[t]{7}{*}{ QLr.cim-2DS } & LR2012Y & 4 & cfd51 & $\operatorname{cfd} 36$ & 5.9 & 12.1 & -4.4 & UC1110 \\
\hline & LR2012AY & 4 & cfd51 & cfd36 & 5.7 & 11.8 & -49.1 & UC1110 \\
\hline & LR2012B & 3 & cfd51 & cfd36 & 13.4 & 26.5 & -11.1 & UC1110 \\
\hline & LR2012AB & 7 & $\operatorname{cfd} 36$ & gwm455 & 13.4 & 26.6 & -109.6 & UC1110 \\
\hline & LR2013B & 7 & $\operatorname{cfd} 36$ & gwm455 & 14.6 & 25.4 & -14.1 & UC1110 \\
\hline & LR2013AB & 6 & $\operatorname{cfd} 36$ & gwm455 & 11.9 & 22.9 & -113.9 & UC1110 \\
\hline & LRM & 7 & $\operatorname{cfd} 36$ & gwm455 & 15.5 & 20.8 & -8.6 & UC1110 \\
\hline \multirow[t]{7}{*}{ QLr.cim-3DC } & LR2012Y & 40 & gwm341 & barc1119 & 4.0 & 8.2 & -4.1 & UC1110 \\
\hline & LR2012AY & 40 & gwm341 & barc1119 & 4.1 & 7.7 & -40.1 & UC1110 \\
\hline & LR2012B & 40 & gwm341 & barc1119 & 6.0 & 11.1 & -5.8 & UC1110 \\
\hline & LR2012AB & 40 & gwm341 & barc1119 & 5.8 & 10.4 & -68.4 & UC1110 \\
\hline & LR2013B & 39 & gwm341 & barc1119 & 10.6 & 17.8 & -11.8 & UC1110 \\
\hline & LR2013AB & 39 & gwm341 & barc1119 & 9.2 & 17.2 & -99.1 & UC1110 \\
\hline & LRM & 39 & gwm341 & barc1119 & 16.9 & 23.3 & -9.2 & UC1110 \\
\hline \multirow[t]{3}{*}{ QYr.cim-3DC } & YR2012M & 40 & gwm341 & barc1119 & 4.5 & 8.9 & -4.6 & UC1110 \\
\hline & YR2012AM & 40 & gwm341 & barc1119 & 3.9 & 7.8 & -28.4 & UC1110 \\
\hline & YRM & 38 & gwm341 & barc1119 & 3.9 & 6.1 & -4.2 & UC1110 \\
\hline \multirow[t]{5}{*}{ QYr.cim-3BS } & YR2012M & 6 & cfb3417 & barc133 & 7.4 & 12.2 & -6.2 & UC1110 \\
\hline & YR2012AM & 6 & cfb3417 & barc133 & 4.9 & 9.8 & -32.0 & UC1110 \\
\hline & YR2013M & 6 & cfb3417 & barc133 & 24.8 & 38.1 & -13.0 & UC1110 \\
\hline & YR2013AM & 6 & cfb3417 & barc133 & 25.6 & 38.2 & -101.8 & UC1110 \\
\hline & YRM & 6 & cfb3417 & barc133 & 25.4 & 33.8 & -13.0 & UC1110 \\
\hline \multirow[t]{5}{*}{ Yr48-5AL } & YR2012M & 183 & wmc727 & gwm410 & 13.2 & 24.7 & 9.4 & PI610750 \\
\hline & YR2012AM & 183 & wmc727 & gwm410 & 7.0 & 15.2 & 44.2 & Pl610750 \\
\hline & YR2013M & 184 & gwm410 & wPt-9800 & 17.2 & 25.1 & 14.1 & PI610750 \\
\hline & YR2013AM & 183 & wmc727 & gwm410 & 17.3 & 23.4 & 84.5 & Pl610750 \\
\hline & YRM & 184 & gwm410 & wPt-9800 & 20.0 & 24.6 & 11.8 & Pl610750 \\
\hline
\end{tabular}

${ }^{a}$ QTL/genes that extend across single one-log support confidence intervals were assigned the same symbol.

intermediate reaction to YR at the seedling stage. Resistance to LR and YR in the UC1110/PI610750 population appears to be controlled by sources of seedling resistance as well as by APR loci. In a previous study, YR resistance in this population was attributed to four APR QTL, including QYr.ucw-2BS and QYr.ucw-3BS derived from UC1110 and QYr.ucw-2AS and Yr48 contributed by PI610750 (Lowe et al., 2011b).

\section{Leaf Rust Seedling Resistance Gene Lr26}

Lr26 is known to be present in the wheat-rye 1RS.1BL chromosome translocation (McIntosh et al., 1995), and this translocation was widely used in wheat breeding programs worldwide during the 1980s and 1990s. For instance, 54\% of the wheat cultivars grown in Bulgaria carry the 1RS.1BL translocation (Landjeva et al., 2006), approximately $38 \%$ of wheat cultivars released since 1980 contain this translocation in China (Zhou et al., 2004), and nearly 50\% of CIMMYT's around 2,000 advanced bread wheat breeding lines were homozygous for the 1RS.1BL translocation in 1988 (Villareal et al., 1991). However, its frequency has decreased to below $5 \%$ in current breeding materials due to the breakdown of resistance genes located on

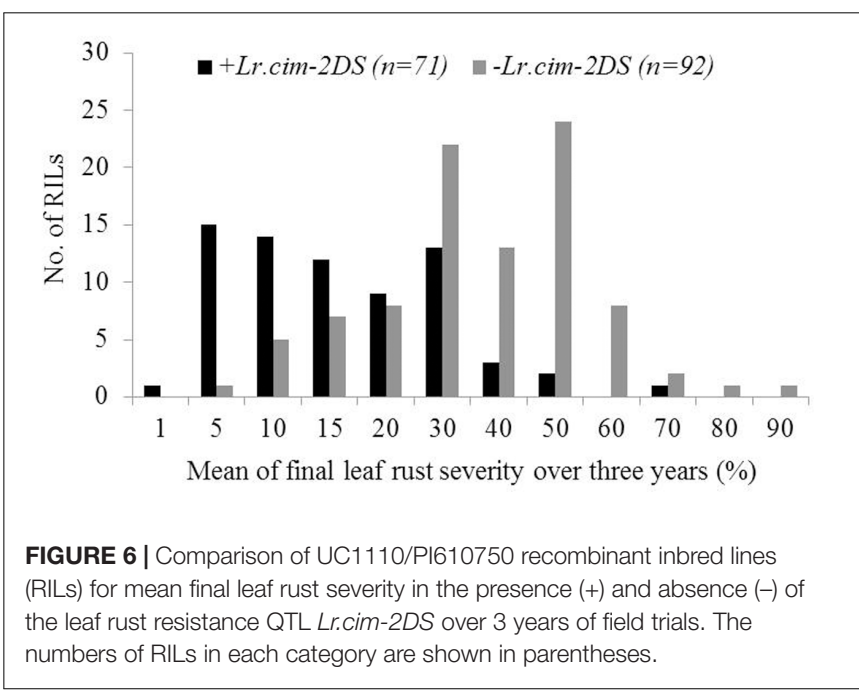

it and to poor quality associated with the translocation. The widespread deployment of the 1RS.1BL translocation was due to the fact that the 1RS arm carries resistance genes to multiple diseases (e.g., $\operatorname{Sr} 31, \mathrm{Yr} 9$, and $\mathrm{Pm} 8$ ) and positively affects various 
TABLE 3 | Two-way factorial analysis of variance (ANOVA) of area under disease progress curve (AUDPC) for leaf rust resistance loci, using years as blocks.

\begin{tabular}{|c|c|c|c|c|c|c|c|}
\hline Source & No. of RILs & DF & Type III SS & Mean square & $\boldsymbol{F}$-value & $P r>F$ & Variation (\%) \\
\hline YEAR & & 2 & 615360.1 & 307680.0 & 10.77 & $<0.0001$ & 3.1 \\
\hline 2DS & 47 & 1 & 3281283.7 & 3281283.7 & 114.81 & $<0.0001$ & 26.1 \\
\hline 3DC & 38 & 1 & 2278573.7 & 2278573.7 & 79.73 & $<0.0001$ & 17.0 \\
\hline $2 \mathrm{DS} \times 3 \mathrm{DC}$ & 33 & 1 & 124795.7 & 124795.7 & 4.37 & 0.0372 & 1.4 \\
\hline
\end{tabular}

TABLE 4 | Three-way factorial analysis of variance (ANOVA) of area under disease progress curve (AUDPC) for stripe rust resistance loci, using years as blocks.

\begin{tabular}{|c|c|c|c|c|c|c|c|}
\hline Source & No. of RILs & DF & Type III SS & Mean square & $F$-value & $P r>F$ & Variation (\%) \\
\hline YEAR & & 1 & 242019.0 & 242019.0 & 28.05 & $<0.0001$ & 0.1 \\
\hline $3 B S$ & 16 & 1 & 1990404.0 & 1990404.0 & 230.73 & $<0.0001$ & 2.8 \\
\hline $5 \mathrm{AL}$ & 11 & 1 & 1158229.5 & 1158229.5 & 134.26 & $<0.0001$ & 16.6 \\
\hline 3DC & 50 & 1 & 211609.8 & 211609.8 & 24.53 & $<0.0001$ & 4.6 \\
\hline $3 B S \times 5 A L$ & 19 & 1 & 727184.1 & 727184.1 & 84.3 & $<0.0001$ & 37.9 \\
\hline $3 B S \times 3 D C$ & 10 & 1 & 0.1 & 0.1 & 0 & 0.998 & 0 \\
\hline $5 \mathrm{AL} \times 3 \mathrm{DC}$ & 28 & 1 & 286.7 & 286.7 & 0.03 & 0.8555 & 0 \\
\hline $3 B S \times 5 A L \times 3 D C$ & 24 & 1 & 11503.6 & 11503.6 & 1.33 & 0.249 & 0.3 \\
\hline
\end{tabular}

agronomic traits (Tang et al., 2008). Unfortunately, races fully virulent on Lr26 were detected in most wheat-growing areas, including Europe, the Indian subcontinent, North America, and South Africa in last middle of 1990s (McIntosh et al., 1995), likely due to the widespread use of a single, major resistance gene over a large area. In the current study, Mexican LR race MBJ/SP was found to be partially virulent on $\operatorname{Lr} 26$, whereas another field race (MCJ/SP) was completely virulent on this gene. Thus, this study was not designed to detect the effect of $\operatorname{Lr} 26$. This gene is closely linked to the YR resistance gene Yr9 (Mago et al., 2005); however, we did not detect the effect of Yr9 because both races used in the field trials were virulent. Lr26 should be contributed by one of PI610750's parents, Kauz (pedigree: Jupateco F73/Bluejay//Ures T81; released in Mexico as Bacanora T88), carrying the 1RS.1BL translocation (Singh and Rajaram, 1991).

\section{Leaf Rust Resistance Locus on 2DS}

The APR QTL for LR, QLr.cim-2DS, contributed by UC1110, is located on the short arm of chromosome 2D, in the 2DS-5 region of the DArT physical map (Francki et al., 2009) and flanked by SSR markers $c f d 36$ and $c f d 51$. So far, five cataloged seedling resistance genes have been detected on the short arm of chromosome 2D, including Lr2 (Ausemus et al., 1946), Lr15 (McIntosh and Baker, 1968), Lr22 (Hiebert et al., 2007), Lr39 (Raupp et al., 2001), and Lr41 (Sun et al., 2009). Lr22 and Lr39 were transferred from A. tauschii, whereas Lr41 was introgressed from Aegilops cylindrica to common wheat. UC1110 (pedigree: Chukar///Yding//Bluebird/Chanate) is an adapted breeding line developed at UC Davis with no indications of these introgressions in its pedigree; therefore, QLr.cim-2DS is expected to be different from these three seedling genes. Lr15 was mapped in the common wheat line "Kenya W1483"; however, the LR races MBJ/SP and $\mathrm{MCJ} / \mathrm{SP}$ are virulent on this gene. Although the two races are avirulent to Lr2, the seedling test suggests that QLr.cim-2DS is not $L r 2$, as the ITs of both parents were " 1 " when challenged with the Lr2-virulent race TCT/QB at the seedling stage. Although several other LR resistance QTL have been mapped in wheat lines, Avocet (Rosewarne et al., 2012), CI 13227 (Xu et al., 2005), and Saar (Zhang et al., 2009) on the distal region of chromosome 2DS. They are located more than $10 \mathrm{cM}$ away from QLr.cim-2DS based on the wheat consensus map (Somers et al., 2004). Thus, it is likely that QLr.cim-2DS is a previously undetected APR locus for LR in common wheat. A single locus mapping population is currently under development to fine map this resistance locus.

\section{Co-located Resistance QTL to both Rusts on 3DC}

The pleiotropic resistance QTL, QLr.cim-3DC/QYr.cim-3DC, was detected near the centromere of chromosome $3 \mathrm{D}$ and flanked by SSR markers gwm341 and barc1119. Basnet et al. (2014) detected QLr.tam-3D/QYr.tam-3D in the CIMMYT wheat line Quaiu\#3 and assigned its location to a proximal region of chromosome arm 3DS, flanked by markers wPt-672034 and barc125. That QTL was found to explain 3-6 and 3.2-4.3\% of the observed variation in LR and YR DS, respectively. The flanking markers for the pleiotropic QTL in this study are only $\sim 4 \mathrm{cM}$ distal of the QLr.tam-3D/QYr.tam-3D locus, based on the wheat consensus map (Somers et al., 2004). QLr.cim-3DC was also detected in an Avocet/Francolin\#1 population (Lan et al., 2014a), where it explained $17.8-25.4 \%$ of the observed variation in LR DS in the Lr46-subtracted sub-population of RILs; but its effect was not significantly detectable on YR. In addition, the pedigree of UC1110 is Chukar/3/Yding//Bluebird/Chanate, while it is Babax/Lr42//Babax*2/3/Vivitsi for Quaiu\#3 (Basnet et al., 2014) and Waxwing*2/Vivitsi for Francolin\#1 (Lan et al., 2014b). The pedigree of Vivitsi is Sonoita F 81/Yaco/5/Bobwhite/Crow//Buckbuck/Pavon F 76/3/Yecora F 70/4/Trap\#1, whereas Bluebird is one of parents for Buckbuck. Therefore, QLr.cim-3DC/QYr.cim-3DC in UC1110 might be the same QTL as in Quaiu\#3 and Francolin\#1 and derived from Bluebird, though a larger effect was observed in UC1110 than in 
either the Quaiu\#3 or Francolin genetic backgrounds. Allelism tests will be required to test these hypotheses.

\section{Stripe Rust Resistance Loci}

One YR APR QTL was mapped in this study to chromosome arm $3 \mathrm{BS}$, within the marker interval $c f d 3417$-barc133, in agreement with the previous study conducted under the California YR environment (Lowe et al., 2011b). In the previous study, the QTL was named QYr.ucw-3BS and was found to be closely linked to gwm533.1, barc133, and wPt-1620. An APR YR resistance gene, Yr30, has also been mapped on the short arm of chromosome 3B (Singh et al., 2000b); however, QYr.ucw-3BS is not Yr30 based on allelism test (Lowe et al., 2011b). While this resistance locus did not offer any significant protection against US YR race PST-100, it did impart partial resistance at the adult plant stage. The IT for UC1110 was "6" against Mexican race Mex14.191, indicating this line might present a minor resistance gene with moderate-susceptible reaction at seedling stage.

The second YR resistance gene, Yr48, was mapped at the same genomic position as reported in the previous study (Lowe et al., 2011b). This resistance gene comes from the synthetic wheat derivative PI610750 and is located in the telomeric region of the long arm of chromosome 5A. Yr48 showed large effect on $\mathrm{YR}$ in the adult plant stage under the U.S. rust environment, with an average reduction of $63 \%$ YR severity compared to fully susceptible lines (Lowe et al., 2011b), whereas it reduced YR severity only up to $37.1 \%$ under the Mexican rust environment. The ITs of RILs with Yr48 ranged from four to six at seedling stage and field severity ranged from 1 to $60 \%$. We conclude that Yr48 confers moderate seedling resistance and APR against the Mexican Pst race used in the study; thus it should be re-classified as an all-stage resistance gene. The segregation frequency of genotypes carrying $\mathrm{Yr} 48$ showed a strong distortion, possibly due to random drift or selection in gametes and/or zygotes. In addition, the genotype of nearby markers for this gene also showed distorted distributions, confirming the YR phenotypic results obtained in seedling.

\section{Interactions between Detected QTL}

Small gene interaction between QLr.cim-2DS and QLr.cim-3DC may explain why the 3DC QTL did not display any significant interactions when combined with other QTL against LR in Avocet/Quaiu\#3 population (Basnet et al., 2014). For YR, the effect of the QYr.ucw-3BS was larger in the presence than in the absence of $Y r 48$, resulting in a highly significant interaction that explained a large proportion of the variation. Lowe et al. (2011b) also reported a highly significant interaction $(P<0.0001)$ between these two QTL with similar effects. No significant interactions were detected between QYr.cim-3DC and other QTL, suggesting that the YR resistance QTL on 3DC had an additive effect.

\section{REFERENCES}

Ausemus, E. R., Harrington, J. B., Reitz, L. P., and Worzella, W. W. (1946). A summary of genetic studies in hexaploid and tetraploid wheats. J. Am. Soc. Agron. 38, 1082-1099.

\section{Application}

UC1110, a high yielding spring wheat line developed by the UC Davis breeding program, provides intermediate resistance to LR and YR in both Mexican and U.S. rust environments. PI610750 remained highly resistant to both rusts in the two countries. We obtained some transgressive RILs combining all of the detected YR resistance QTL, such as RILs 8, 26, 28, 119, and 254, with DS lower than 1\% across Mexican and U.S. rust environments, whereas RILs 26 and 184 carry two LR resistance QTL resulting in DS of approximately $2 \%$. We used two RILs (43 and 170) carrying the single resistance QTL 2DS and crossed them with RIL 180 (no resistance QTL) to develop single gene mapping populations for fine mapping and further characterization of this locus. The closely linked molecular markers to QLr.cim-2DS (within 0.5-2.3 cM), QYr.ucw-3BS (within $0.2 \mathrm{cM}$ ), and Yr48 (within 0.6-0.7 cM) can be immediately used in wheat breeding programs to improve rust resistance via marker-assisted selection.

\section{AUTHOR CONTRIBUTIONS}

CL phenotyped the population, did analysis, and wrote the manuscript. IH and JD genotyped the population with DArT and SSR markers. SH-F, BB, MR, JH-E, and RS phenotyped the population at both seedling and adult plant stages.

\section{FUNDING}

This work was supported by the Australian Grains Research and Development Corporation (GRDC), the Australian Cereal Rust Control Program (ACRCP), and the National Natural Science Foundation of China (31301309). Dubcovsky acknowledges support from the National Research Initiative Competitive Grant 2011-68002-30029 (Triticeae-CAP) from the USDA National Institute of Food and Agriculture.

\section{ACKNOWLEDGMENT}

We appreciate the English editing by Julie Mollins.

\section{SUPPLEMENTARY MATERIAL}

The Supplementary Material for this article can be found online at: http://journal.frontiersin.org/article/10.3389/fpls.2017.01450/ full\#supplementary-material

Basnet, B. R., Singh, R. P., Ibrahim, A. M. H., Herrera-Foessel, S. A., HuertaEspino, J., Lan, C. X., et al. (2014). Characterization of Yr54 and other genes associated with adult plant resistance to yellow rust and leaf rust in common wheat Quaiu 3. Mol. Breed. 33, 385-399. doi: 10.1007/s11032-0139957-2 
Bjarko, M. E., and Line, R. F. (1988). Heritability and number of genes controlling leaf rust resistance in four cultivars of wheat. Phytopathology 78, 457-461. doi: 10.1094/Phyto-78-457

Chen, X. M. (2005). Epidemiology and control of stripe rust (Puccinia striiformis f. sp. tritici) on wheat. Can. J. Plant Pathol. 27, 314-337. doi: 10.1080/ 07060660509507230

Chen, X. M. (2013). High-temperature adult-plant resistance, key for sustainable control of stripe rust. Am. J. Plant Sci. 4, 608-627. doi: 10.4236/ajps.2013.43080

Chen, X. M. (2014). Stripe Rust: The Good, Bad and Ugly. Pullman, DC: Washington Grain Commission, 52-55.

Das, M. K., Rajaram, S., Mundt, C. C., Kronstad, W. E., and Singh, R. P. (1992). Inheritance of slow rusting resistance in wheat. Crop Sci. 32, 1452-1456. doi: $10.2135 /$ cropsci1992.0011183X003200060028x

Flor, H. H. (1942). Inheritance of pathogenicity in Melampsora lini. Phytopathology 32, 653-669.

Francki, M. G., Walker, E., Crawford, A. C., Broughton, S., Ohm, H. W., Barclay, I., et al. (2009). Comparison of genetic and cytogenetic maps of hexaploid wheat (Triticum aestivum L.) using SSR and DArT markers. Mol. Genet. Genomics 281, 181-191. doi: 10.1007/s00438-008-0403-9

Herrera-Foessel, S. A., Singh, R. P., Lillemo, M., Huerta-Espino, J., Bhavani, S., Singh, S., et al. (2014). Lr67/Yr46 confers adult plant resistance to stem rust and powdery mildew in wheat. Theor. Appl. Genet. 127, 781-789. doi: 10.1007/ s00122-013-2256-9

Hiebert, C. W., Thomas, J. B., Somers, D. J., McCallum, B. D., and Fox, S. L. (2007). Microsatellite mapping of adult-plant leaf rust resistance gene $\operatorname{Lr} 22 a$ in wheat. Theor. Appl. Genet. 115, 877-884. doi: 10.1007/s00122-007-0604-3

Hovmøller, M. S., Walter, S., Bayles, R. A., Hubbard, A., Flath, K., Sommerfeldt, N., et al. (2015). Replacement of the European wheat yellow rust population by new races from the centre of diversity in the near-Himalayan region. Plant Pathol. 65, 402-411. doi: 10.1111/ppa.12433

Huang, B. E., George, A. W., Forrest, K. L., Kilian, A., Hayden, M. J., Morell, M. K., et al. (2012). A multiparent advanced generation inter-cross population for genetic analysis in wheat. Plant Biotechnol. J. 10, 826-839. doi: 10.1111/j. 1467-7652.2012.00702.x

Huerta-Espino, J., Villaseñor-Mir, H. E., Rodriguez-Garcia, M. F., and Singh, R. P. (2015). "Emerging new virulence gene combinations in the Mexican Pst population," in Proceedings of the BGRI 2015 Technical Workshop 17-20 September, Sydney, NSW.

Johnson, R., and Law, C. N. (1973). Cytogenetic studies on the resistance of the wheat variety Bersée to Puccinia striiformis. Cereal Rusts Bull. 1, 38-43.

Jones, J. D. G., and Dangl, J. L. (2006). The plant immune system. Nature 444, 323-329. doi: 10.1038/nature05286

Knott, D. R., and Padidam, M. (1988). Inheritance of resistance to stem rust in six wheat lines having adult plant resistance. Genome 30, 283-288. doi: 10.1139/ g88-049

Krattinger, S. G., Lagudah, E. S., Spielmeyer, W., Singh, R. P., Huerta-Espino, J., McFadden, H., et al. (2009). A putative ABC transporter confers durable resistance to multiple fungal pathogens in wheat. Science 323, 1360-1362. doi: 10.1126/science. 1166453

Lan, C. X., Rosewarne, G. M., Singh, R. P., Herrera-Foessel, S. A., Huerta-Espino, J., Basnet, B. R., et al. (2014a). QTL characterization of resistance to leaf rust and stripe rust in the spring wheat line Francolin\#1. Mol. Breed. 34, 789-803. doi: 10.1007/s11032-014-0075-6

Lan, C. X., Singh, R. P., Huerta-Espino, J., Calvo-Salazar, V., and Herrera-Foessel, S. A. (2014b). Genetic analysis of resistance to leaf rust and stripe rust in wheat cultivar Francolin\# 1. Plant Dis. 98, 1227-1234. doi: 10.1094/PDIS-07-130707-RE

Landjeva, S., Korzun, V., Tsanev, V., Vladova, R., and Ganeva, G. (2006). Distribution of the wheat-rye translocation 1RS.1BL among bread wheat varieties of Bulgaria. Plant Breed. 125, 102-104. doi: 10.1111/j.1439-0523.2006. 01142.x

Li, Z. F., Lan, C. X., He, Z. H., Singh, R. P., Rosewarne, G. M., Chen, X. M., et al. (2014). Overview and application of QTL for adult plant resistance to leaf rust and powdery mildew in wheat. Crop Sci. 54, 1907-1925. doi: 10.2135/ cropsci2014.02.0162

Lowe, I., Cantu, D., and Dubcovsky, J. (2011a). Durable resistance to the wheat rusts: integrating systems biology and traditional phenotype-based research methods to guide the deployment of resistance genes. Euphytica 179, 69-79.
Lowe, I., Jankuloski, L., Chao, S. M., Chen, X. M., See, D., and Dubcovsky, J. (2011b). Mapping and validation of QTL which confer partial resistance to broadly virulent post-2000 North American races of stripe rust in hexaploid wheat. Theor. Appl. Genet. 123, 143-157. doi: 10.1007/s00122-011-1573-0

Maccaferri, M., Zhang, J., Bulli, P., Abate, Z., Chao, S., Cantu, D., et al. (2015). A genome-wide association study of resistance to stripe rust (Puccinia striiformis $\mathrm{f}$. sp. tritici) in a worldwide collection of hexaploid spring wheat (Triticum aestivum L.). G3 5, 449-465. doi: 10.1534/g3.114.014563

Mago, R., Miah, H., Lawrence, G. J., Wellings, C. R., Spielmeyer, W., Bariana, H. S., et al. (2005). High-resolution mapping and mutation analysis separate the rust resistance gene $\operatorname{Sr} 31, \operatorname{Lr} 26, \operatorname{Yr} 9$ on the short arm of rey chromosome 1. Theor. Appl. Genet. 112, 41-50. doi: 10.1007/s00122-005-0098-9

McIntosh, R. A., and Baker, E. P. (1968). “A linkage map for chromosome 2D," in Proceedings of the 3rd International Wheat Genetics Symposium, eds K. W. Finlay and K. W. Shepard (Sydney, NSW: Butterworth \& Co), 305-308.

McIntosh, R. A., Dubcovsky, J., Rogers, W. J., Morris, C., Appels, R., and Xia, X. C. (2016). Catalogue of Gene Symbols for Wheat: 20152016 (Supplement). Available at: https://shigen.nig.ac.jp/wheat/komugi/genes/ macgene/supplement2015.pdf

McIntosh, R. A., Hart, G. E., and Gale, M. D. (1995). "Catalogue of gene symbols for wheat," in Proceedings of the 8th International Wheat Genetics Symposium, eds Z. S. Li and Z. Y. Xin (Beijing: China Agricultural Scientech Press), 1333-1500.

McNeal, F. H., Konzak, C. F., Smith, E. P., Tate, W. S., and Russell, T. S. (1971). A Uniform System for Recording and Processing Cereal Research Data. Washington, DC: Agricultural Research Service, 34-121.

Meng, L., Li, H. H., Zhang, L. Y., and Wang, J. K. (2015). QTL IciMapping: integrated software for genetic linkage map construction and quantitative trait locus mapping in biparental populations. Crop J. 3, 269-283. doi: 10.1016/j.cj. 2015.01.001

Moore, J. W., Herrera-Foessel, S., Lan, C. X., Schnippenkoetter, W., Ayliffe, M., Huerta-Espino, J., et al. (2015). A recently evolved hexose transporter variant confers resistance to multiple pathogens in wheat. Nat. Genet. 47, 1494-1498. doi: 10.1038/ng.3439

Peterson, R. F., Campbell, A. B., and Hannah, A. E. (1948). A dia-grammatic scale for estimating rust intensity on leaves and stems of cereals. Can. J. Res. 26, 496-500. doi: 10.1139/cjr48c-033

Raupp, W. J., Singh, S., Brown-Guedira, G. L., and Gill, B. S. (2001). Cytogenetic and molecular mapping of the leaf rust resistance gene Lr39 in wheat. Theor. Appl. Genet. 102, 347-352. doi: 10.1007/s001220051652

Roelfs, A. P., Singh, R. P., and Saari, E. E. (1992). Rust Diseases of Wheat: Concepts and Methods of Disease Management. Mexico: CIMMYT.

Rosewarne, G. M., Herrera-Foessel, S. A., Singh, R. P., Huerta-Espino, J., Lan, C. X., and He, Z. H. (2013). Quantitative trait loci of stripe rust resistance in wheat. Theor. Appl. Genet. 126, 2427-2449. doi: 10.1007/s00122-013-2159-9

Rosewarne, G. M., Singh, R. P., Huerta-Espino, J., Herrera-Foessel, S. A., Forrest, K. L., Hayden, M. J., et al. (2012). Analysis of leaf and stripe rust severities reveals pathotype changes and multiple minor QTLs associated with resistance in an Avocet $\times$ Pastor wheat population. Theor. Appl. Genet. 124, 1283-1294. doi: $10.1007 / \mathrm{s} 00122-012-1786-\mathrm{x}$

Seyfarth, R. (2000). Development of Molecular Markers for the Adult Plant Leaf Rust Resistance Genes Lr13 and Lr35 in Wheat (Triticum aestivum L.). Ph.D. thesis, Swiss Federal Institute of Technology, Zürich.

Singh, R. P., Herrera-Foessel, S. A., Huerta-Espino, J., Bariana, H., Bansal, U., McCallum, B., et al. (2012). "Lr34/Yr18/Sr57/Pm38/Bdv1/Ltn1 confers slow rusting, adult plant resistance to Puccinia graminis tritici," in Proceedings of the 13th International Cereal Rusts and Powdery Mildews Conference, ed. W.-Q. Chen (Beijing: Friendship Hotel).

Singh, R. P., Herrera-Foessel, S. A., Huerta-Espino, J., Lan, C. X., Basnet, B. R., Bhavani, S., et al. (2013). "Pleiotropic gene Lr46/Yr29/Pm39/Ltn2 confers slow rusting, adult plant resistance to wheat stem rust fungus," in Proceedings of the 2013 Technical Workshop: Borlaug Global Rust Initiative, New Delhi.

Singh, R. P., Huerta-Espino, J., and Rajaram, S. (2000a). Achieving near-immunity to leaf and stripe rusts in wheat by combining slow rusting resistance genes. Acta Phytopathol. Hung. 35, 133-139.

Singh, R. P., Nelson, J. C., and Sorrells, M. E. (2000b). Mapping Yr28 and other genes for resistance to stripe rust in wheat. Crop Sci. 40, 1148-1155. doi: 10.1094/PHYTO-03-15-0060-R 
Singh, R. P., and Rajaram, S. (1991). Resistance to Puccinia recondita f. sp. tritici in 50 Mexican bread wheat cultivars. Crop Sci. 31, 1472-1479. doi: 10.2135/ cropsci1991.0011183X003100060016x

Somers, D. J., Isaac, P., and Kdwards, K. (2004). A high-density microsatellite consensus map for bread wheat (Triticum aestivum L.). Theor. Appl. Genet. 109, 1105-1114. doi: 10.1007/s00122-004-1740-7

Sun, X. C., Bai, G. H., and Carver, B. F. (2009). Molecular markers for wheat leaf rust resistance gene Lr41. Mol. Breed. 23, 311-321. doi: 10.1007/s11032-0089237-8

Tang, Z. X., Fu, S. L., Ren, Z. L., Zhang, H. Q., Yang, Z. J., Yan, B. J., et al. (2008). Production of a new wheat cultivar with a different 1B.1R translocation with resistance to powdery mildew and stripe rust. Cereal Res. Commun. 36, 451-460. doi: 10.1556/CRC.36.2008.3.9

Todorovska, E., Christov, N., Slavov, S., Christova, P., and Vassilev, D. (2009). Biotic stress resistance in wheat-breeding and genomic selection implications. Biotechnol. Biotechnol. Equip. 23, 1417-1426. doi: 10.2478/V10133-009-0006-6

van Ooijen, J. W. (2006). Join Map 4, Software for the Calculation of Genetic Linkage Maps in Experimental Population. Wageningen: Plant Research International.

Vazquez, D. M., Zemetra, R., Peterson, J. C., Chen, X. M., Heesacker, A., and Mundt, C. C. (2015). Multi-location wheat stripe rust QTL analysis: genetic background and epistatic interactions. Theor. Appl. Genet. 128, 1307-1318. doi: 10.1007/s00122-015-2507-z

Villareal, R. L., Rajaram, S., Mujeeb-Kazi, A., and Del Toro, E. (1991). The effect of chromosome $1 \mathrm{~B} / 1 \mathrm{R}$ translocation on the yield potential of certain spring wheats (Triticum aestivum L.). Plant Breed. 106, 77-81. doi: 10.1111/j.1439-0523.1991. tb00482.x

Voorrips, R. E. (2002). MapChart: software for the graphical presentation of linkage maps and QTLs. Heredity 93, 77-78. doi: 10.1093/jhered/93.1.77
Wang, S. C., Basten, J., and Zeng, Z. B. (2012). Windows QTL Cartographer 2.5. Raleigh, NC: North Carolina State University.

Wilkinson, P. A., Winfield, M. O., Barker, G. L. A., Allen, A. M., Burridge, A., Coghill, J. A., et al. (2012). CerealsDB 2.0: an integrated resource for plant breeders and scientists. BMC Bioinformatics 13:219. doi: 10.1186/1471-210513-219

Xu, X. Y., Bai, G. H., Carver, B. F., Shaner, G. E., and Hunger, R. M. (2005). Mapping of QTLs prolonging the latent period of Puccinia triticina infection in wheat. Theor. Appl. Genet. 110, 244-251. doi: 10.1007/s00122-0041819-1

Zhang, L., Li, Z. F., Lillemo, M., Xia, X. C., Liu, D. Q., Yang, W., et al. (2009). QTL mapping for adult-plant resistance to leaf rust in CIMMYT wheat cultivar Saar. Sci. Agric. Sin. 42, 388-397.

Zhou, Y., He, Z. H., Zhang, G. S., Xia, L. Q., Chen, X. M., Gao, Y. C., et al. (2004). Utilization of 1BL/1RS translocation in wheat breeding in China. Acta Agron. Sin. 30, 531-535.

Conflict of Interest Statement: The authors declare that the research was conducted in the absence of any commercial or financial relationships that could be construed as a potential conflict of interest.

Copyright (c) 2017 Lan, Hale, Herrera-Foessel, Basnet, Randhawa, Huerta-Espino, Dubcovsky and Singh. This is an open-access article distributed under the terms of the Creative Commons Attribution License (CC BY). The use, distribution or reproduction in other forums is permitted, provided the original author(s) or licensor are credited and that the original publication in this journal is cited, in accordance with accepted academic practice. No use, distribution or reproduction is permitted which does not comply with these terms. 